\title{
Screening of halogenated phenolic compounds in plasma and serum from marine wildlife
}

\author{
D. Lindquist ${ }^{1}$ (i)
}

Received: 12 July 2019 / Revised: 22 October 2019 / Accepted: 18 December 2019 / Published online: 31 December 2019

(c) The Author(s) 2019

\begin{abstract}
The growing knowledge of the impact of halogenated phenolic compounds on hormonal and metabolic systems has led to an increased interest in the exposure and potential effects of these compounds in wildlife. In the present study, a screening procedure was developed to detect and quantify halogenated phenolic compounds in serum and plasma from marine wildlife. A mass spectral library containing selective ion monitoring data was created using gas chromatography electron capture negative ionization mass spectrometry. The selective ion monitoring data in the library were accompanied with retention indices to increase the specificity of each entry in the library. The library together with the developed extraction procedure and optimized instrumental settings can be used for the detection of 52 different halogenated phenolic compounds of environmental concern, including 23 hydroxylated polychlorinated biphenyls and 24 hydroxylated polybrominated diphenyl ethers. The instrument limit of detection for the compounds included in the library ranged from 30 to $320 \mathrm{fg} / \mathrm{injection}$, with a median detection limit of $90 \mathrm{fg} /$ injection. The average recovery of 11 different halogenated phenolic compounds, from four species of marine wildlife, was $66 \pm 14 \%$. A full-scan mass spectral library was also created containing an additional seven compounds. Gray seals, long-tailed ducks, and two species of fish from the Baltic Sea were screened for halogenated phenolic compounds using the developed procedure. A total of 33 compounds included in the library were detected and quantified.
\end{abstract}

Keywords Analysis $\cdot$ Blood $\cdot$ Phenols $\cdot$ Mass spectral library $\cdot$ Retention index

\section{Introduction}

Halogenated organic compounds (HPCs) are of environmental concern due to their ubiquity in nature and biological activity. Chlorophenols, in particular pentachlorophenol (PCP), have been used extensively as biocides for the protection of wood, which has led to a near-global contamination. PCP is a non-specific biocide that affects oxidative phosphorylation thus making it toxic to humans as well as wildlife (Weinbach 1957).

Editorial responsibility: M. Abbaspour.

Electronic supplementary material The online version of this article (https://doi.org/10.1007/s13762-019-02607-w) contains supplementary material, which is available to authorized users.

D. Lindqvist

dennis.lindqvist@aces.su.se

1 Department of Environmental Science and Analytical Chemistry, Stockholm University, Stockholm, Sweden
The chlorinated phenoxyphenol triclosan has also been spread widely through its use as an antibacterial agent in personal care products. Triclosan started to increase in use, and thus in environmental levels during the 1970s. The release of triclosan to the environment decreased somewhat toward the 1980s following increased establishment of wastewater treatment plants, but soon started to increase again during the 1990s as a result of increased popularity (Singer et al. 2002). Exposure to environmentally relevant concentrations of triclosan has been observed to affect development in zebrafish (Danio rerio) (Stenzel et al. 2019). Triclosan also affects the lipid metabolism and causes liver toxicity (Huang et al. 2019).

Chlorinated phenolic compounds are also formed via metabolic hydroxylation of polychlorinated biphenyls (PCBs) resulting in OH-PCBs (Jansson et al. 1975). OHPCBs can cause adverse effects through interaction with the estrogen receptor (Ruiz et al. 2016), as well as by affecting the thyroid hormone system (Dirinck et al. 2016) and mitochondrial functions (Narasimhan et al. 1991). 
Brominated phenolic compounds have been released to the environment via the use of brominated flame retardants (BFRs). Some BFRs are phenolic in their native configuration such as tetrabromobisphenol A (TBBPA), which has been shown to alter expression reproductive-relevant genes in fish (von Krogh et al. 2019). Other BFRs may be converted to HPCs as a result of metabolism (Hakk and Letcher 2003).

Brominated phenolic compounds of an impressively diverse variety are also produced naturally by bacteria, fungi, and algae (Gribble 2010). In the Baltic Sea, the levels of some of these brominated compounds, specifically hydroxylated polybrominated diphenyl ethers (OH-PBDE), have been observed to become particularly high during the summer months in species such as, e.g., perch (Perca fluviatilis) (Dahlgren et al. 2016). Like PCP, most of the dominant OH-PBDEs in the Baltic Sea have been determined to affect oxidative phosphorylation (Legradi et al. 2017). OH-PBDEs have also been observed to be genotoxic (Ji et al. 2011), neurotoxic (Dingemans et al. 2008), endocrine disruptive (Li et al. 2013; Yu et al. 2015), and to cause developmental malformations in fish (Liu et al. 2015).

Blood plasma and serum are suitable matrices for determining the exposure to HPCs. Blood can be accessed from many species without having to sacrifice the specimen and HPCs accumulated in blood due to their high affinity for plasma proteins (Louis et al. 2015), such as serum albumin (Yang et al. 2017). However, plasma and serum analyses of HPCs have been proven to be less than trivial and large discrepancies have been observed related to the quality of (amount of hemolysis in) the samples (Dahlberg et al. 2014). Recently hemin, ferric heme released from hemoglobin, was identified as the main causative agent behind poor recoveries and precision during blood analysis of HPCs (Lindqvist and Asplund 2019). Understanding the discrepancies observed during blood analysis of HPCs enables the development of more robust analytical methods for HPCs in serum and plasma samples.

The detection and quantification of HPCs in the environment are routinely done by gas chromatography electron capture negative ionization mass spectrometry (GC-ECNI/ MS). GC-ECNI/MS generally holds an edge over electron impact (EI) MS in terms of selectivity and sensitivity for halogenated compounds, hence enabling the detection of lower levels. However, mass spectral information gained by ECNI/MS is often inferior to that gained by EI/MS and exciting MS libraries are not as evolved for ECNI/MS as for EI/MS. Liquid chromatography mass spectrometry methods have also been developed for the determination of HPCs in biological samples (Quinete et al. 2015; Hu et al. 2017).

The aim of this study was divided into three parts. The first was to develop and evaluate a method for extracting HPCs from a variety of serum and plasma samples from different species and of different quality. The requirements on the method were that it should be simple, it should negate the influence of hemin on the recovery of HPCs, and it should provide small variations in recovery over species and type of HPC. The second part was to build a MS library with included retention indices that could be used for identification of HPCs even in selected ion monitoring (SIM) mode, which would allow for screening of small sample volumes. The third part was to apply the extraction method to screen plasma and serum from a few Baltic Sea animals for HPCs using the MS library together with automatic deconvolution software. By automatizing the data handling, the analysis time can be significantly decreased. The study was conducted throughout 2017-2018 at Stockholm University (Stockholm, Sweden).

\section{Materials and methods}

\section{Chemicals and reagents}

Reference standards were purchased from Wellington Laboratories (Guelph, ON, Canada) or synthesized in-house. Sources for each of the reference chemicals are listed in the electronic supplementary material (ESM), Table S1 in ESM_1. Ethanol (99.7\%) was acquired from Sovelco (Rosersberg, Sweden). Isohexane (IHX) and water for HPLC, as well as toluene (Tol) formic acid (99-100\%) and diethyl ether (DEE) of ACS grade, from BDH chemicals were purchased from VWR International (Radnor, PA, USA). Methanol for HPLC and 2,2,4-trimethylpentane (TMP) for GC were acquired from Merck (Darmstadt, Germany). Tetramethylammonium hydroxide (TMAOH; $25 \%$ in methanol), $N, N$-diisopropylethylamine (Hünig's base; $\geq 98 \%$ ), and methyliodide ( $\geq 99 \%$ ) for GC were all from Sigma-Aldrich (Saint Louis, MO, USA).

\section{Samples}

All gray seal blood (Halichoerus grypus) was collected during the inventory of the seal population and monitoring of seal health in the Baltic Sea, within the national environmental monitoring program in Sweden. The samples were collected during the spring of 2012 and 2013 mainly from Bothnia Bay. All of the seal blood was donated from the Swedish Museum of Natural History. Long-tailed duck blood (Clangula hyemalis), collected at Åland in May of 2010, was the remains from previous studies. Blood from these birds was only used for method evaluation, and no quantitative data for HPCs have been published (Dahlberg et al. 2014). However, quantitative data for HPCs in the livers from the long-tailed ducks have been published (Dahlberg et al. 2016). Herring (Clupea harengus) and flounder 
(Platichthys flesus) plasma collected at the Askö marine research station in May and June of 2011 were also remains from previous studies; no data have been published on these samples. All blood collections were conducted with proper ethical permits at the time of the sampling, and no additional permits were necessary for this study. Information on how the blood was collected from the fishes and ducks can be found in Dahlberg et al. (2014). All samples were stored at $-20{ }^{\circ} \mathrm{C}$ prior to usage. Storage over longer periods at $-20{ }^{\circ} \mathrm{C}$ may cause some deterioration of the serum plasma matrix. However, the HPCs are stable at these temperatures over long periods as long as they are stored in the dark.

\section{Analytical procedure}

\section{Extraction}

To $1 \mathrm{~g}$ of serum or plasma, formic acid/ethanol $(3: 1,4 \mathrm{~mL})$ and IHX/Tol (3:1, $4 \mathrm{~mL})$ were added. The sample was mixed vigorously and centrifuged. The organic phase was isolated, and the acid phase was re-extracted with IHX/Tol $(3: 1,1 \mathrm{~mL})$. The two organic fractions were pooled together.

\section{Cleanup}

Water $(2 \mathrm{~mL})$ was added to the organic fraction. The sample was mixed and centrifuged, and the water phase was discarded. Potassium hydroxide in $50 \%$ ethanol $(0.5 \mathrm{M}, 3 \mathrm{~mL})$ was then added, and the sample was mixed to isolate the HPCs in the alkaline phase. The organic phase was discarded (note that this fraction contains, e.g., PCBs and PBDEs if their detection should be of interest). Aqueous citric acid $(50 \%, 1 \mathrm{~mL})$ was added to acidify the alkaline phase, and the HPCs were back-extracted into IHX/diethyl ether (3:1, $4 \mathrm{~mL}$ ).

\section{Derivatization}

Methanol $(200 \mu \mathrm{L})$ was added to the organic fraction before reducing the volume until only a few droplets remained using a stream of nitrogen gas. TMAOH in methanol (5\%, 20 $\mu \mathrm{L})$ was added as well as Hünig's base $(20 \mu \mathrm{L})$. Iodomethane $(200 \mu \mathrm{L})$ was then added, and the solution was incubated for $1 \mathrm{~h}$ at $50^{\circ} \mathrm{C}$ (ensure that the test tubes are gas tight before incubation to avoid risk of exposure to iodomethane).

After the reaction was complete, the excess reagent was evaporated off, again until only a few $\mu \mathrm{L}$ of the solution remained. The sample was resolved in IHX $(2 \mathrm{~mL})$ and TMP $(1 \mathrm{~mL})$. The solution was mixed with $\mathrm{KOH}$ in $50 \%$ ethanol $(0.5 \mathrm{M}, 1 \mathrm{~mL})$ to clean the extract, remove tetramethylammonium iodide, and quench any remaining iodomethane. The $\mathrm{KOH}$ phase was discarded after centrifugation. (A small amount of water, $\sim 0.5 \mathrm{~mL}$, may be added to increase surface tension and make the phase separation more visible if needed.) The sample was then finally treated with concentrated sulfuric acid $(2 \mathrm{~mL})$. The volume of the organic phase was then adjusted by evaporation under a stream of nitrogen gas to approximately $100 \mu \mathrm{L}$ before injection on GC-MS.

\section{Recovery experiment}

One of the main requirements of the screening method was that it should be applicable to a large variety of marine wildlife. Hence, several species were included in the method evaluation. The test species included one mammal (gray seal) one marine bird species (long-tailed duck), and two species of fish, one benthic (flounder), and one pelagic species (herring). The method was also evaluated for both plasma and serum with varying amounts of hemolysis. The samples were divided into three groups based on visual observation, little hemolysis, average hemolysis, and plenty of hemolysis (an example picture can be found in ESM_1, Fig. S1). The samples were spiked in two levels $1 \mathrm{ng} / \mathrm{g}$ and $5 \mathrm{ng} / \mathrm{g}$. The effect of pausing the analytical work after the extraction and continuing with the cleanup the next day was also tested. The test compounds were HPCs belonging to different groups, including: bromophenols (BPs), OH-PBDEs, OH-PCBs, and TBBPA. In total, 11 compounds were tested. The specific compounds chosen for the evaluation were such that were expected to occur only at very low concentrations or be absent in the serum and plasma. The test compound mixture was dissolved in methanol $(100 \mu \mathrm{L})$ when spiked to the serum and plasma. The samples were let to equilibrate for at least $2 \mathrm{~h}$ before extractions were commenced. BDE139 was added as a volumetric standard before instrumental analysis, and the recovery of each compound was calculated against a three-point calibration curve.

\section{Mass spectral library setup}

A full-scan and an SIM ECNI/MS library (included as supplementary material, ESM_2_Full and ESM_3_SIM) were created for halogenated phenolic compounds derivatized by methylation. The libraries were created using NISTs freeware AMDIS (Automated Mass spectral Deconvolution and Identification System), which allows for the use of retention indices for identification. In the SIM library, two of the dominant $\mathrm{m} / \mathrm{z}$ produced in the MS for each analyte were generally included for identification, together with the retention index (on a DB-35MS capillary column). A total of 59 compounds were included in the libraries (52 for SIM) including 27 (23) OH-PCBs, 27 (24) OH-PBDEs, and five miscellaneous (2,4,6-tribromophenol, pentachlorophenol, triclosan, TBBPA, and 2,2'-diOH-BB80) (see Table S1 in ESM_1). One of the included OH-PCBs (4-OH-CB193) 
and OH-PBDEs (4'-OH-BDE121), respectively, were used as surrogate standards within the quantitative part of the study. The SIM analyses were divided into eight time-segments, and at the most four $\mathrm{m} / \mathrm{z}$ were monitored in each of these SIM windows (see Table S1 in ESM_1). Information on how to use the libraries and retention indices for identification can be found in the ESM_1 together with suggested software settings.

\section{Screening of Baltic Sea wildlife}

The same four species that were used to evaluate the extraction procedure were also analyzed to determine the native content and levels of the HPCs included in the SIM database. The analyses were conducted as a first application to test the screening procedure as a whole. Seven individual gray seal specimens including males, females, and juveniles were analyzed. Three long-tailed duck samples were analyzed, a female, a male, and a male juvenile. Each duck sample was in turn a pool from two individual specimens. One flounder and one herring sample were analyzed. Both fish samples were pools from approximately 15 individuals each of mixed gender. In total, 12 samples were analyzed. Each sample was extracted in triplicates of $1 \mathrm{~g}$ that were pooled before instrumental analyses.

Quantifications were conducted using AMDIS, by making two copies of the SIM MS library and introducing the proper response factors for 4-OH-CB193 and 4'-OH-BDE121 into each of the copies. (These copies are not included as supporting information, but the response factor gained on the instrument for each analyte can be found in Table S1 in ESM_1.) Each detected analyte was quantified using both surrogates, and the average value was determined.

\section{Instrumental settings}

All analyses were conducted on an Agilent 7890A GC (Agilent Technologies) with a multimode injector (MMI), coupled to a 5975C MS. The MS was operated in ECNI mode for the detection of methylated HPCs, and in positive ion chemical ionization (PICI) mode for alkanes (used to calculated retention indices). A $5 \mu \mathrm{L}$ aliquot was injected in liquid vent mode at $75{ }^{\circ} \mathrm{C}$. The solvent was vented at 5 psi for $0.04 \mathrm{~min}$. The injector was then ramped in two stages: first to $255^{\circ} \mathrm{C}$ (held for $1.86 \mathrm{~min}$ ) and then to $325^{\circ} \mathrm{C}$, both times at $600{ }^{\circ} \mathrm{C} / \mathrm{min}$. The split was opened after $2.2 \mathrm{~min}(2.4 \mathrm{~min}$ for alkanes) with a purge flow of $60 \mathrm{~mL} / \mathrm{min}$. The septum purge was $3 \mathrm{~mL} / \mathrm{min}$. Helium $(1.25 \mathrm{~mL} / \mathrm{min})$ was used as the mobile gas. The $\mathrm{GC}$ oven was programmed from $65^{\circ} \mathrm{C}$ (held for $2.2 \mathrm{~min}$ ) to $250^{\circ} \mathrm{C}$ at $25^{\circ} \mathrm{C} / \mathrm{min}$, then to 285 at $5{ }^{\circ} \mathrm{C} / \mathrm{min}$, and finally to $310^{\circ} \mathrm{C}$ at $25^{\circ} \mathrm{C} / \mathrm{min}$ (held for $5.4 \mathrm{~min}$ ). The whole program was $23 \mathrm{~min}$. A J\&W DB-35MS UI capillary column was used $(20 \mathrm{~m} \times 0.18 \mathrm{~mm}$ i.d. $\times 0.18 \mu \mathrm{m}$ film thickness; J\&W Scientific, CA, USA). The transfer line to the MS was set at $310^{\circ} \mathrm{C}$, and the ion source temperature was $200{ }^{\circ} \mathrm{C}$. Methane was used as buffer gas for ECNI/MS and as reagent gas for PICI/MS, with vacuum pressures of $\sim 1.9 \mathrm{E}-04$ torr and $\sim 7.0 \mathrm{E}-05$ torr, respectively.

\section{Results and discussion}

\section{Method development}

\section{Extraction and recoveries}

The extraction of seal plasma and serum produced the lowest recoveries of all included species with an average recovery of $64 \%$ (for 11 compounds over 9 extractions, $n=11 \times 9$ ), which can be compared to $70 \%$ for long-tailed duck $(n=11 \times 4), 69 \%$ for flounder $(11 \times 2)$, and $70 \%$ for herring $(11 \times 2)$. The seal samples seemed to yield a slightly higher recovery when extracting serum (average recovery $65.5 \%, n=11 \times 5)$ compared to plasma $(62 \%, n=11 \times 4)$, although there was no statistical significance ( $p=0.14$, twosided $t$ test) (see Fig. 1; for the full dataset, see Table S2 in ESM_1). No significant difference $(p=0.30)$ could be observed between long-tailed-duck serum $(67 \%, n=11 \times 2)$ and plasma $(72 \%, 11 \times 2)$ either, only plasma was available for fish. The recoveries of the more heavily brominated $\mathrm{OH}$ PBDEs (especially penta and hexa) seemed to be a bit better in the long-tailed duck and fish compared to the seal samples. On the other hand, the recovery of the tribrominated OH-PBDE (6'-OH-BDE17) was better in the seal samples. For the included OH-PCBs and TBBPA, there seemed to be

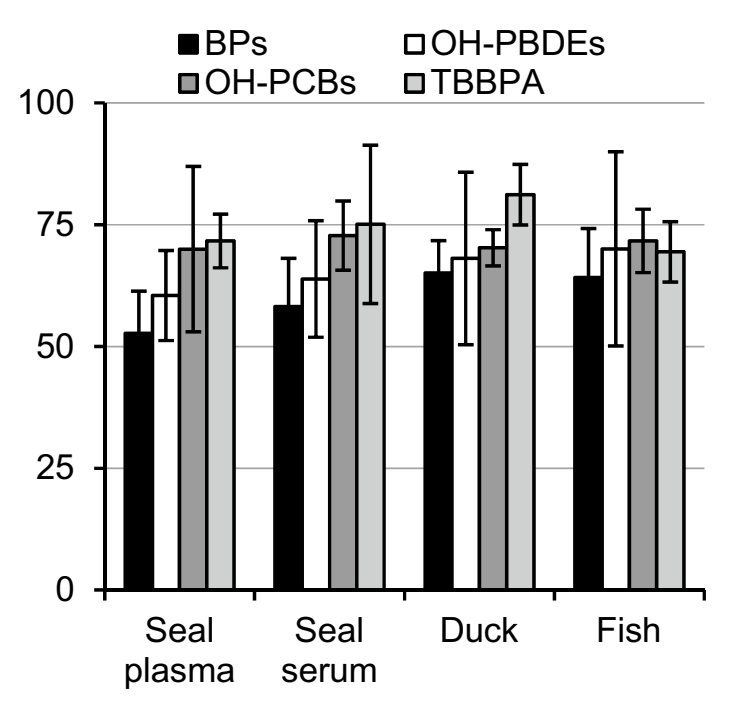

Fig. 1 Average recovery of different groups of HPCs from seal, longtailed duck and fish. Full dataset included in the supplementary material (Table S2 in ESM_1) 
no apparent differences. The simple bromophenols produced the lowest recoveries as a group, with the tribromophenol $(2,3,5$-TBP) producing lower recoveries $(52 \%, n=17)$ than the tetrabromophenol $(2,3,4,6-\mathrm{TTBP})(67 \%, n=17)$, which may be an indication of losses related to vapor pressure (i.e., losses during evaporation steps) (see Fig. 1 and Table S2 in ESM_1). The amount of hemolysis in the serum and plasma had no effect on the recovery using the developed method. The average recovery of HPCs from samples with little hemolysis was $66 \%(n=11 \times 9)$, average amount of hemolysis $68 \%(n=11 \times 3)$, and plenty of hemolysis $66 \%$ $(n=11 \times 5)$. There was no statistically significant difference in recovery with regard to hemolysis in any of the tested species. No difference could be observed based on the concentration of the spiked compounds except for in the seal, where the lower concentration actually produced significantly $(p=0.009)$ higher recoveries $(68 \%, n=11 \times 3)$ than the higher concentration $(62 \%, n=11 \times 6)$.

Furthermore, there was no statistically significant difference $(p=0.15)$ in whether cleanup was commenced directly following the extraction (average recovery $67 \%, n=11 \times 12$ ), or if the extract was left in the refrigerator overnight before continuing the analysis (average recovery $64 \%, n=11 \times 5$ ) (see Table S2 in ESM_1). Previous studies have shown that leaving OH-PBDEs for longer periods in non-polar solvents, in the presence of hemin, may lead to decreased recoveries (Lindqvist and Asplund 2019). Because of the effect of the solvents polarity on the suggested complex formation between OH-PBDEs and hemin, the analysis was paused before washing the extracts with water when the samples were left overnight (see Materials and methods). By doing so, a small portion of formic acid was left in the extracts, which increased the polarity of the solutions. The same principle was evoked when adding the small amount of methanol before reducing the volume of the samples in preparation for the derivatization.

The extraction method presented here was largely developed to separate HPCs from hemin. It was observed that hemin distributed mainly to the formic acid phase when it was partitioned between formic acid and toluene, while the HPCs distributed to the toluene phase. Since hemin was considered to be the most problematic matrix component, the extraction was based on this principle. As toluene by itself solvated a quite large amount of formic acid, it was diluted with TMP in the method, to decrease co-extraction of formic acid. As the water from the serum/plasma decreased the solubility of hemin in the acid phase, an equal amount of ethanol was added, thus resulting in the extraction mixtures 3:1 TMP/toluene and 3:1 formic acid/ethanol. The TMP/Tol extract was still washed with a small amount of water prior to $\mathrm{pH}$ partitioning, as mentioned, to remove any co-extracted acid. It was also observed that exchanging hydrochloric acid for citric acid during back-extraction of HPCs into organic solvents after the $\mathrm{pH}$ partitioning increased the solubility of potential co-extracted hemin in the water/ethanol phase.

If whole blood is to be analyzed, the use of formic acid is not to be recommended as it facilitates the release of heme from hemoglobin, which increases the formation of hemin in the sample as previously discussed (Lindqvist and Asplund 2019).

\section{Derivatization}

To avoid the use of diazomethane, a procedure for methylation of HPCs using iodomethane was developed. Note that there are still risks associated with the derivatization procedure describe here and that great caution should be taken. Iodomethane is a well-established reagent for methylation reactions. Here, a small amount of TMAOH in methanol was used to initially charge the HPCs. The excess TMAOH reacts rapidly with iodomethane, which is added in a much larger excess, leading to formation of methanol and precipitation of tetramethylammonium iodide. Because of the rapid neutralization of the TMAOH, a small amount of Hünig's base was added to act as a proton scavenger during the reaction. The reaction was conducted at $50{ }^{\circ} \mathrm{C}$ to increase the reactivity. The reaction was limited by the nucleophilic strength of the phenolate ions of the HPCs, hence HPCs with lower $\mathrm{pK}_{a}$ values tended to react the slowest. However, after $1 \mathrm{~h}$ the yields were on par with that of diazomethane for all tested compounds. Care should be taken before applying this method to other types of matrices as it could not be used, as is, when large amounts of free fatty acids were present, without a significant drop in yield.

\section{GC-MS analysis}

Trace analysis of environmental toxins does not lend itself readily to full-scan mass spectrometric detection of analytes, at least in regard to low resolution MS. For halogenated compounds, a great enhancement in signal-to-noise $(\mathrm{S} / \mathrm{N})$ can usually be achieved when progressing from GC-EI/MS to GC-ECNI/MS, and sometimes full-scan analyses are possible. However, for brominated organic compounds low-level analyses tend to yield mostly bromide ions in ECNI, thus limiting the possibility to secure a positive identification using a mass spectral library. The use of retention indices in combination with mass spectral information can greatly improve the chance to secure an accurate positive identification when conducting a library search. In this study, even SIM data were used in combination with retention indices to identify target analytes. The ability to apply SIM for accurate identification was of course largely enabled by combining the instrumental analysis with a selective extraction and work-up procedure that target compounds with certain 
polarity, acidity, and stability toward concentrated sulfuric acid following methylation.

Only four $\mathrm{m} / \mathrm{z}$ were monitored at any given time during the SIM analysis, which enabled the use of moderate dwell times without loss of scan rate (i.e., the number of times each ion is measured per time unit) and subsequently resolution in the chromatograms (i.e., the number of data points per time unit in the chromatogram). It further produces good $\mathrm{S} / \mathrm{N}$ ratios for the analytes in the total ion chromatogram (TIC) and overall low detection limits, which allows for analysis of low levels. The instrument limit of detection (ILOD; set to five times the level of the noise in the TIC) for the HPCs included in the SIM library ranged from 30 to $320 \mathrm{fg} /$ injection, with a median ILOD of $90 \mathrm{fg} / \mathrm{injection}$ (see Table S1 in ESM_1).

\section{Screening of Baltic Sea wildlife}

A total of 33 individual HPCs included in the SIM database were detected during the screening of Baltic Sea wildlife. Seven HPCs were detected in all of the samples: PCP, 2,4,6-tribromophenol (2,4,6-TBP), 2 OH-PCBs (4-OH$\mathrm{CB} 187$ and 4-OH-CB163), and 3 OH-PBDEs (6-OHBDE47, 6-OH-BDE90, and 2'-OH-BDE68). 4-OH-CB146 and 4-OH-CB177 were detected in all species except in the flounder.

At an average concentration of $41 \mathrm{ng} / \mathrm{g}$ wet weight (w.w.), 4-OH-CB 107 was by far the most abundant $\mathrm{OH}-\mathrm{PCB}$ in the gray seal serum and plasma, where it made up on average $84 \%$ of the $\Sigma \mathrm{OH}-\mathrm{PCB}$ concentration (see Fig. 2 and Table S3 in ESM_1). 4-OH-CB107 may be formed by direct insertion of an $\mathrm{OH}$-group in $\mathrm{CB} 107$, but it can also be formed via 1,2-shift following epoxidation of the more prevalent PCB congener CB105 and CB118. The second most abundant OH-PCB in the seal was 4-OH-CB162 (8\%), which like 4-OH-CB 107 can be formed by direct insertion of an OH-group in CB162 or by 1,2-shift following epoxidation of $\mathrm{CB} 157$ or $\mathrm{CB} 167$. The third most abundant congener was 4'-OH-CB120 (2\%), which can be formed from CB120 or once again from CB118. 3-OH-CB118 was also detected in all seal samples but in lower concentration. All three of the most dominant $\mathrm{OH}-\mathrm{PCB}$ congeners (making up $94 \%$ of the $\Sigma \mathrm{OH}-\mathrm{PCB}$ concentration) stem from metabolization of mono-ortho-chlorinated PCBs. The semi-planar mono-ortho-PCBs are potential substrates for the oxidative enzyme CYP1A1, and hence the dominance of mono-ortho$\mathrm{OH}-\mathrm{PCB}$ in gray seals may stem from a high activity of this enzyme. 4-OH-CB107 has previously been reported as the dominant HPC in Baltic Sea gray seals (Lindqvist and Asplund 2019) as well as in northern elephant seals (Louis et al. 2015), and its dominance among OH-PCBs in ringed seals (Phoca hispida) as a result of its metabolic formation via CYP1A1 has previously been discussed (Routti et al. 2008).

In both fish species, as well as in the long-tailed duck, $p$-chlorophenyl-tetrachlorophenol-type $\mathrm{OH}-\mathrm{PCB}$ (see Fig. 3), including 4-OH-CB187 (dominant), 4-OH-CB163, 4-OH-CB177, and 4-OH-CB178, were instead the most abundant (see Table S3 in ESM_1). Together, these congeners made up $77 \%, 93 \%$, and $100 \%$ of the $\Sigma \mathrm{OH}-\mathrm{PCB}$ concentration in long-tailed duck, herring, and flounder, respectively. The fifth most abundant OH-PCB congener in seals and third most abundant in long-tailed duck and herring was 4-OH-CB146. 4-OH-CB146 was expected to be of

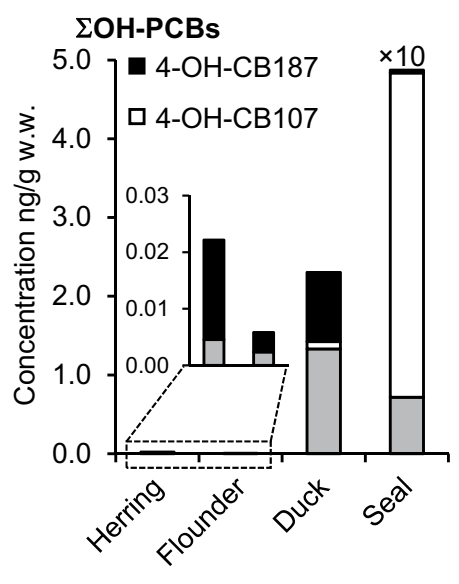

Fig. 2 Levels of different HPCs detected in the four studied species. (Left) $\Sigma O H-P C B$ concentration in serum/plasma from four different species (in ng/g w.w.) contribution from 4-OH-CB187 and 4-OHCB107 to the total concentration shown in black and white, respectively, all other congeners are included in the gray portion. Note that the seal levels have been scaled down one order of magnitude. (Mid-

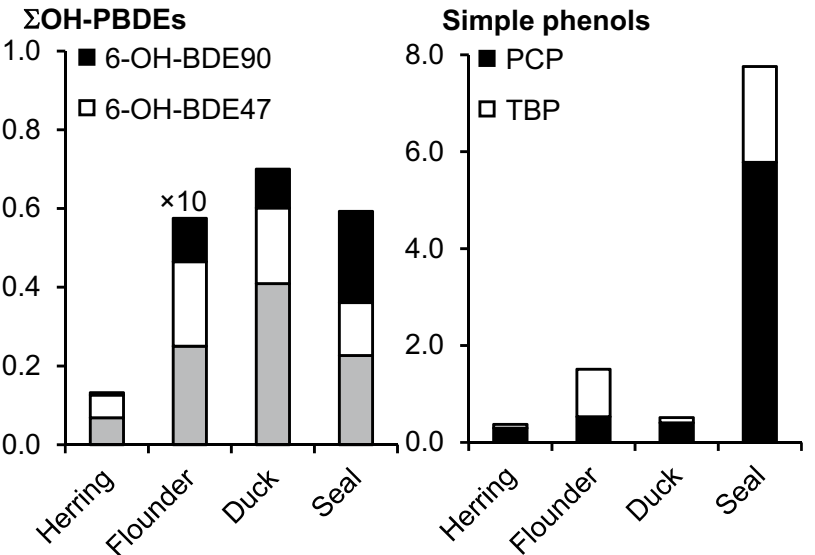

dle) $\Sigma \mathrm{OH}-\mathrm{PBDE}$ concentration, contribution from 6-OH-BDE90 and 6-OH-BDE47 to the total concentration shown in black and white, respectively. Note that the flounder levels have been scaled down one order of magnitude. (Right) concentration of PCP and 2,4,6-TBP. Full dataset included in ESM_1 (Table S3 and Table S4) 


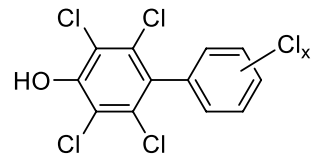

Fig. 3 Common structure for the $p$-chlorophenyl-tetrachlorophenoltype $\mathrm{OH}-\mathrm{PCB}$. This structure dominated the $\mathrm{OH}-\mathrm{PCB}$ exposure in the two fish species as well as in the long-tailed duck

high concentration as it may stem from metabolization (via 1,2-shift) of two of the most abundant PCBs in Baltic Sea biota, CB153 and CB138. 3-OH-CB153 was indicated in all seal and long-tailed duck samples, but no quantitative data are reported due to low concentrations (high uncertainty). 3'-OH-CB138 was the fourth most abundant OH-PCB congener in seals (see Table S3 in ESM_1).

6-OH-BDE47 was the most abundant OH-PBDE congener overall (see Fig. 2). However, a slightly higher average level of 6-OH-BDE90 was recorded in seal (see Fig. 2) and slightly higher average level of 6-OH-BDE99 was recorded in long-tailed duck (see Table S4 in ESM_1). All of these OH-PBDEs have been suggested to be produced natural by filamentous algae, in the Baltic Sea (Lindqvist et al. 2017).

Gray seals had the highest exposure of HPCs, and the exposure was dominated by OH-PCBs. Both long-tailed duck and flounder feed on blue mussels in the Baltic Sea and thus are more likely to be exposed to naturally produced $\mathrm{OH}$ PBDEs. Flounder was indeed the most OH-PBDE exposed species with a $\Sigma \mathrm{OH}-\mathrm{PBDE}$ plasma concentration of nearly $6 \mathrm{ng} / \mathrm{g}$ w.w., which was three orders of magnitude higher than the level of $\Sigma O H-P C B$ (made up by only two detected congeners however) (see Fig. 2 and Tables S3 and S4 in ESM_1). Long-tailed ducks on the other hand seem to possess a much greater ability to accumulate and/or metabolize PCBs resulting in a higher level of $\Sigma \mathrm{OH}-\mathrm{PCBs}(2.3 \mathrm{ng} / \mathrm{g}$ w.w.) than of $\Sigma$ OH-PBDEs ( $0.7 \mathrm{ng} / \mathrm{g}$ w.w.) (see Fig. 2 and Tables S3 and S4 in ESM_1).

\section{Conclusion}

The method presented here should only be considered as a semi-quantitative screening method. Manual integration and quantification against an external calibration curve were made for a few samples, and it did indicate that the automatic quantification made in AMDIS produces satisfactory results, generally within $\pm 30 \%$ of the results gained by external calibration. The response factors need to be continuously updated, and external calibration needs to be run on a regular basis to assess linearity of the instrument, to ensure the quality of the quantifications. To truly assess the accuracy of the presented method, it will be necessary to take part in intercalibration studies in the future. Until then, the extraction and cleanup method presented here together with the MS libraries do seem to provide a good tool for screening purposes. Note that ECNI is not as robust an ionization technique as EI, and small variations in fragmentation can occur between instruments, depending on the type of ion source that is used. In the future, it may be interesting to use the large set of retention indices recorded for methylated $\mathrm{OH}-$ PCBs and OH-PBDEs in this study (Table S1 in ESM_1) to develop a quantitative structure-retention relationship (QSRR) model, similar to what was recently done for PCBs (Yali et al. 2017). Having a QSRR model to estimate the retention index of other congeners within these homologous groups, on a DB-35 column, could aid in tentatively identifying other congeners in environmental samples. During the screening part of this study, interesting structural differences were observed among the major OH-PCB congeners in the different species, which may be an indication of a large difference in the metabolism of PCBs among marine wildlife.

Acknowledgements Open access funding provided by Stockholm University. Olle Karlsson at the Swedish Museum of Natural History is greatly acknowledged for collecting and donating gray seal plasma and serum. Kjell Larsson at the Kalmar Maritime Academy, Linnaeus University, is acknowledged for collecting the long-tailed duck samples and allowing us to use them for this study. Also, thanks to Anna-Karin Dahlberg at the Swedish University of Agricultural Science (SLU) for her contribution in collecting fish samples and to Ioannis Athanassiadis at ACES Stockholm University for valuable discussion related to the mass spec databases.

\section{Compliance with ethical standards}

Conflict of interest The author declares that he has no conflict of interest.

Open Access This article is licensed under a Creative Commons Attribution 4.0 International License, which permits use, sharing, adaptation, distribution and reproduction in any medium or format, as long as you give appropriate credit to the original author(s) and the source, provide a link to the Creative Commons licence, and indicate if changes were made. The images or other third party material in this article are included in the article's Creative Commons licence, unless indicated otherwise in a credit line to the material. If material is not included in the article's Creative Commons licence and your intended use is not permitted by statutory regulation or exceeds the permitted use, you will need to obtain permission directly from the copyright holder. To view a copy of this licence, visit http://creativecommons.org/licenses/by/4.0/.

\section{References}

Dahlberg A-K, Norrgran J, Hovander L, Bergman Å, Asplund L (2014) Recovery discrepancies of OH-PBDEs and polybromophenols in human plasma and cat serum versus herring and long-tailed duck plasma. Chemosphere 94:97-103

Dahlberg A-K, Lindberg Chen V, Larsson K, Bergman Å, Asplund L (2016) Hydroxylated and methoxylated polybrominated diphenyl ethers in Long-tailed duck (Clangula hyemalis) and their main 
food, Baltic blue mussels (Mytilus trossulus x Mytilus edulis). Chemosphere 144:1475-1483

Dahlgren E, Lindqvist D, Dahlgren H, Asplund L, Lehtilä K (2016) Trophic transfer of naturally produced brominated aromatic compounds in a Baltic Sea food chain. Chemosphere 144:1597-1604

Dingemans MML, de Groot A, van Kleef RGDM, Bergman $\AA$, van den Berg M, Vijverberg HPM, Westerink RHS (2008) Hydroxylation increases the neurotoxic potential of BDE-47 to affect exocytosis and calcium homeostasis in PC12 cells. Environ Health Perspect 116:637-643

Dirinck E, Dirtu AC, Malarvannan G, Covaci A, Jorens PG, Van Gaal LF (2016) A preliminary link between hydroxylated metabolites of polychlorinated biphenyls and free thyroxin in humans. Int $\mathrm{J}$ Environ Res Public Health 13:421-433

Gribble GW (2010) Naturally occurring organohalogen compounds-a comprehensive update. Prog Chem Org Nat Prod 91:1-613

Hakk H, Letcher RJ (2003) Metabolism in the toxicokinetics and fate of brominated flame retardants-a review. Environ Int 29:801-828

Hu D, Hu X, Chen W, Wang H, Wang H, Zhu K, Wu B, Lin C (2017) Determination of hydroxylated polybrominated diphenyl ethers in Chinese aquatic products by LC-MS/MS. J Chrom Sci 55:918-925

Huang W, Xie P, Cai Z (2019) Lipid metabolism disorders contribute to hepatotoxicity of triclosan in mice. J Hazard Mater. https://doi. org/10.1016/j.jhazmat.2019.121310

Jansson B, Jensen S, Olsson M, Renberg L, Sundström G, Vaz R (1975) Identification by GC-MS of phenolic metabolites of PCB and p, p'-DDE isolated from Baltic guillemot and seal. Ambio 4:93-97

Ji K, Choi K, Giesy JP, Musarrat J, Takeda S (2011) Genotoxicity of several polybrominated diphenyl ethers (PBDEs) and hydroxylated PBDEs, and their mechanisms of toxicity. Environ Sci Technol 45:5003-5008

Legradi J, van Pomeren M, Dahlberg AK, Legler J (2017) Effects of hydroxylated polybrominated diphenyl ethers in developing Zebrafish are indicative of disruption of oxidative phosphorylation. Int J Mol Sci 18:970-982

Li X, Gao Y, Guo LH, Jiang G (2013) Structure-dependent activities of hydroxylated polybrominated diphenyl ethers on human estrogen receptor. Toxicology 309:15-22

Lindqvist D, Asplund L (2019) Determination of hydroxylated polybrominated diphenyl ethers in blood from Baltic grey seals. J Anal Test. https://doi.org/10.1007/s41664-019-00084-1

Lindqvist D, Dahlgren E, Asplund L (2017) Biosynthesis of hydroxylated polybrominated diphenyl ethers and the correlation with photosynthetic pigments in the red alga Ceramium tenuicorne. Phytochemistry 133:51-58

Liu H, Tang S, Zheng X, Zhu Y, Ma Z, Liu C, Hecker M, Saunders DMV, Giesy JP, Zhang X, Yu H (2015) Bioaccumulation, biotransformation, and toxicity of BDE-47, 6-OH-BDE-47, and
6-MeO-BDE-47 in early life-stages of zebrafish (Danio rerio). Environ Sci Technol 49:1823-1833

Louis C, Covaci A, Stas M, Crocker DE, Malarvannan G, Dirtu AC, Debier C (2015) Bioaccumulation of hydroxylated polychlorinated biphenyls and pentachlorophenol in the serum of northern elephant seal pups (Mirounga angustirostris). Environ Res 136:441-448

Narasimhan TR, Kim HL, Safe SH (1991) Effects of hydroxylated polychlorinated-biphenyls on mouse-liver mitochondrial oxidative-phosphorylation. J Biochem Toxicol 3:229-236

Quinete N, Kraus T, Belov VN, Aretz C, Esser A, Schettgen T (2015) Fast determination of hydroxylated polychlorinated biphenyls in human plasma by online solid phase extraction coupled to liquid chromatography-tandem mass spectrometry. Anal Chim Acta 888:94-102

Routti H, Letcher RJ, Arukwe A, van Bavel B, Yoccoz NG, Chu S, Gabrielsen GW (2008) Biotransformation of PCBs in relation to phase I and II xenobiotic-metabolizing enzyme activities in ringed seals (Phoca hispida) from Svalbard and the Baltic Sea. Environ Sci Technol 42:8952-8958

Ruiz P, Ingale K, Wheeler JS, Mumtaz M (2016) 3D QSAR studies of hydroxylated polychlorinated biphenyls as potential xenoestrogens. Chemosphere 144:2238-2246

Singer H, Muller S, Tixier C, Pillonel L (2002) Triclosan: occurrence and fate of a widely used biocide in the aquatic environment: field measurements in wastewater treatment plants, surface waters, and lake sediment. Environ Sci Technol 36:4998-5004

Stenzel A, Wirt H, Patten A, Theodore B, King-Heiden T (2019) Larval exposure to environmentally relevant concentrations of triclosan impairs metamorphosis and reproductive fitness in zebrafish. Reprod Toxicol 87:79-86

von Krogh K, Ropstad E, Nourizadeh-Lillabadi R, Haug TM, Weltzien F-A (2019) In vitro effects of bisphenol a and tetrabromobisphenol a on cell viability and reproduction-related gene expression in pituitaries from sexually maturing Atlantic cod (Gadus morhua L.). Fishes 4:48-63

Weinbach EC (1957) Biochemical basis for the toxicity of pentachlorophenol. Proc Natl Acad Sci USA 43:393-397

Yali ZP, Jadid AP, Samin LA (2017) Modeling of retention time for polychlorinated biphenyl congeners in human adipose tissue using quantitative structure-retention relationship methodology. Int J Environ Sci Technol 14:2357-2366

Yang L, Yang W, Wu Z, Yi Z (2017) Binding of hydroxylated polybrominated diphenyl ethers with human serum albumin: spectroscopic characterization and molecular modeling. Luminescence 32:978-987

Yu H, Wondrousch D, Li F, Chen J, Lin H, Ji L (2015) In silico investigation of the thyroid hormone activity of hydroxylated polybrominated diphenyl ethers. Chem Res Toxicol 28:1538-1545 